\title{
Nonlinear wave effects at the non-reflecting beach
}

\author{
I. Didenkulova ${ }^{1,2}$ and E. Pelinovsky ${ }^{3,4}$ \\ ${ }^{1}$ Laboratory of Wave Engineering, Institute of Cybernetics at Tallinn University of Technology, Akadeemia tee 21, \\ 12618 Tallinn, Estonia \\ ${ }^{2}$ Nizhny Novgorod State Technical University, Nizhny Novgorod, Russia \\ ${ }^{3}$ Department of Nonlinear Geophysical Processes, Institute of Applied Physics, Nizhny Novgorod, Russia \\ ${ }^{4}$ Department of Information System, Higher School of Economics, Nizhny Novgorod, Russia
}

Correspondence to: I. Didenkulova (ira@cs.ioc.ee)

Received: 8 October 2011 - Revised: 3 December 2011 - Accepted: 5 December 2011 - Published: 3 January 2012

\begin{abstract}
Nonlinear effects at the bottom profile of convex shape (non-reflecting beach) are studied using asymptotic approach (nonlinear WKB approximation) and direct perturbation theory. In the asymptotic approach the nonlinearity leads to the generation of high-order harmonics in the propagating wave, which result in the wave breaking when the wave propagates shoreward, while within the perturbation theory besides wave deformation it leads to the variations in the mean sea level and wave reflection (waves do not reflect from "non-reflecting" beach in the linear theory). The nonlinear corrections (second harmonics) are calculated within both approaches and compared between each other. It is shown that for the wave propagating shoreward the nonlinear correction is smaller than the one predicted by the asymptotic approach, while for the offshore propagating wave they have a similar asymptotic. Nonlinear corrections for both waves propagating shoreward and seaward demonstrate the oscillatory character, caused by interference of the incident and reflected waves in the second-order perturbation theory, while there is no reflection in the linear approximation (firstorder perturbation theory). Expressions for wave set-up and set-down along the non-reflecting beach are found and discussed.
\end{abstract}

\section{Introduction}

Propagating in the real ocean waves normally lose its energy because of numerous reflections from coasts and any inhomogeineities of the seabed (Massel, 1989; Mei, 1989; Dean and Dalrymple, 2002). These effects have been demonstrated in numerical simulations (Kowalik and Murty, 1993; Yeh et al., 1996; Liu et al., 2008) and measured in the coastal zone (Raubenneimer and Guza, 1996; Raubenneimer et al., 2001; Dean and Walton, 2009). Theoretically, wave propagation and reflection in a basin of variable depth are usually analyzed within the linear theory framework; such analytical solutions can be found in books by (Massel, 1989; Mei, 1989; Dean and Dalrymple, 2002). Probably, the only case studied rigorously in the framework of nonlinear shallowwater theory is the wave propagation along the constant slope beach (Carrier and Greenspan, 1958; Synolakis, 1987; Didenkulova et al., 2008). It has been shown that the wave becomes steeper and breaks while climbing the beach. The wave spectrum near the coast becomes wide demonstrating considerable contribution of high-harmonics. For the arbitrary varying bottom profile the study of nonlinear effects during wave propagation becomes very difficult. It requires solving nonlinear PDEs with variable coefficients, while even in the first-order (linear) case the solution has a complicated form.

However, for certain bottom geometries the structure of the solution found within the linear theory has a simple form and can be expressed in elementary functions. For example, in some special cases ("non-reflecting configurations") the wave can propagate along rapidly changing seabed geometry without reflection up to the coast. Such non-reflecting configurations have been found and studied for different bottom geometries. In 1-D case they are represented by two convex beaches $h \sim x^{4 / 3}$ and $h \sim x^{4}$ (Didenkulova et al., 2009; Didenkulova and Pelinovsky, 2010). A number of different non-reflecting configurations have been found for narrow bay and channels (Didenkulova and Pelinovsky, 2011). For all these geometries, except the only case of inclined channel of a parabolic cross-section (Didenkulova and Pelinovsky, 2009), the linear problem has been considered. Amplitude of the wave, propagating along such non-reflecting configurations varies according to the Green's law and tends to infinity at the shoreline. This extreme wave amplification may be reduced by nonlinear effects, which lead to the wave breaking. In this paper we study nonlinear effects of wave propagation at the non-reflecting beach $h \sim x^{4 / 3}$. 


\section{Nonlinear wave transformation in a basin of slowly varying depth}

The dynamics of long waves (in the nearshore region all waves can be considered as long) can be described by the nonlinear shallow water theory

$\frac{\partial \eta}{\partial t}+\frac{\partial}{\partial x}[(h+\eta) u]=0 \quad \frac{\partial u}{\partial t}+u \frac{\partial u}{\partial x}+g \frac{\partial \eta}{\partial x}=0$,

where $\eta(x, t)$ is water displacement, $u(x, t)$ is depthaveraged velocity, $h(x)$ is an arbitrary unperturbed water depth, $g$ is gravity acceleration, $x$ is a coordinate directed offshore, and $t$ is time.

Here we analyze the wave propagation along the beach profile

$h(x)=h_{0}\left[\frac{x}{x_{0}}\right]^{4 / 3}$.

This profile can be often observed in natural conditions. Its existence was demonstrated in (Didenkulova et al., 2009, 2010; Didenkulova and Soomere, 2011) for different geographic locations. Here we underline the work by (Didenkulova and Soomere, 2011), where the beach changes were studied with respect to the joint effect of wind and ship waves in the Baltic Sea. It was shown that during the field experiment the equilibrium beach kept the non-reflecting shape Eq. (2) for any wave conditions.

In the linear shallow water approximation the beach profile Eq. (2) allows non-reflecting wave propagation even for significant bottom slopes (Didenkulova et al., 2009). In order to study nonlinear effects along this beach, at first approximation we assume smooth and slow depth variations $(d h / d x \ll 1)$. In this case the reflected wave should be weak even for the nonlinear problem. Since the energy flux is conserved in smoothly inhomogeneous medium, further we will call this case adiabatic or WKB approach. The nonlinear transformation of weakly nonlinear long waves in the basin of slowly varying depth is studied in (Varley et al., 1971; Gurtin, 1975; Caputo and Stepanyants, 2003; Didenkulova, 2009) within asymptotic nonlinear WKB approximation for different kinds of bottom profiles. In the case of the weakamplitude wave above the non-reflecting beach Eq. (2) it can be described by the following expression in the implicit form

$$
\begin{aligned}
& \eta(T, x)= \\
& =A_{0}\left(\frac{x_{0}}{x}\right)^{1 / 3} \eta_{0}\left(T+\frac{9 \eta x_{0}}{8 h_{0} \sqrt{g h_{0}}}\left[\left(\frac{x_{0}}{x}\right)^{4 / 3}-1\right]\left(\frac{x}{x_{0}}\right)^{1 / 3}\right),
\end{aligned}
$$

where $A_{0}$ and $\eta_{0}$ are amplitude and shape of the water displacement in the point $x=x_{0}$, which is assumed to be far from the coast, $h_{0}$ is a depth at the point $x=x_{0}$, $T=t-\tau(x)$ is time in the reference system of coordinates, $\tau(x)=\int_{x_{0}}^{x} d x / \sqrt{g h(x)}$ is a travel time from the point $x=x_{0}$.
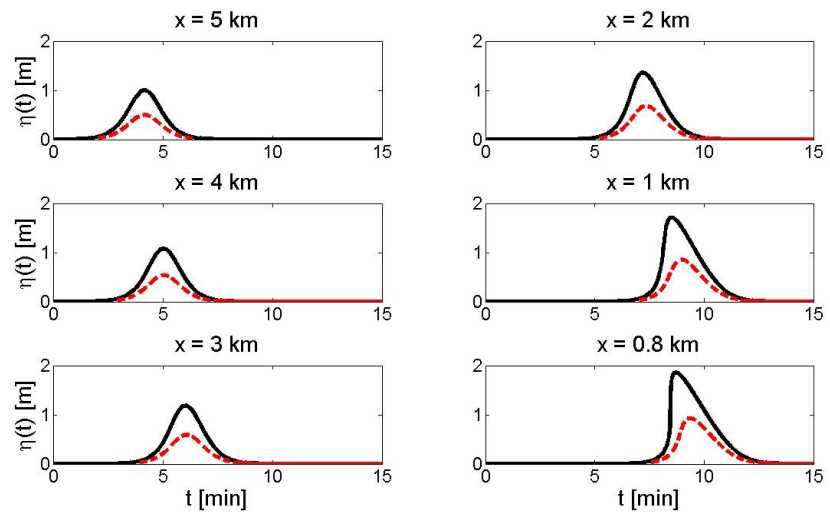

Fig. 1. Time series of nonlinear wave transformation during its propagation to the coast; red dashed line corresponds to the wave with $A_{0}=0.5 \mathrm{~m}$, black solid line - to $A_{0}=1 \mathrm{~m}$.

The details of the derivation of Eq. (3) can be found in (Didenkulova, 2009). Equation (3) describes the wave propagating in any onshore or offshore direction. In the case of the wave propagating to the shore, it can be seen from Eq. (3) that the wave increases its amplitude and steepens during the propagation to the coast. The demonstration of this process is shown in Fig. 1 for initial solitary wave with different amplitudes, located at $x_{0}=5 \mathrm{~km}$ at the depth $h_{0}=40 \mathrm{~m}$

$\eta_{0}(t)=A_{0} \cosh ^{-2}\left(t / T_{0}\right)$,

where $T_{0}=60 \mathrm{~s}$ is the characteristic duration of the wave.

The wave of $1 \mathrm{~m}$ height steepens faster than the one of $0.5 \mathrm{~m}$ height and breaks at $780 \mathrm{~m}$ far from the coast, while the $0.5-\mathrm{m}$ wave breaks $480 \mathrm{~m}$ from the coast. The breaking distance (the distance from the coast, where the wave front becomes vertical) can also be found from Eq. (3) (Didenkulova, 2009). In the case of the non-reflecting beach Eq. (2) it can be re-written as:

$X_{\mathrm{Br}}=\frac{x_{0}}{\left[1+\frac{8 h_{0} \sqrt{g h_{0}}}{9 A_{0} x_{0} \max \left(\eta_{0}^{\prime}\right)}\right]^{3 / 4}}$,

where the prime means derivative with respect to the argument of $\eta_{0}$ in Eq. (3). For a sine wave Eq. (5) has a form

$X_{\mathrm{Br}}=\frac{x_{0}}{\left[1+\frac{8 h_{0} \sqrt{g h_{0}}}{9 A_{0} \omega x_{0}}\right]^{3 / 4}}$.

It follows from Eq. (6) that the wave propagating onshore always breaks.

It should be noted, that hyperbolic equations consider only plunging breaking, which is in agreement with numerous observation of wave breaking near the coast. Spilling breakers, characterized by convective wave breaking should be described by models of the next level, for example, in the Boussinesq approximation (Sorensen, 1993; Sato and Kabiling, 1995; Nwogu, 1996; Bjorkavag and Kalisch, 2011). 
For the wave propagating seawards $\left(x>x_{0}\right)$ the sign of the second term in argument of the function $\eta_{0}$ in Eq. (3) will be opposite and correspondingly the second term in the denominator of the breaking distance Eqs. (5) and (6) should have an opposite sign. As a result, the wave propagating offshore can break only if its amplitude is unrealistically large

$A_{0} \geq \frac{8 h_{0} \sqrt{g h_{0}}}{9 x_{0} \max \left(\eta_{0}^{\prime}\right)}$.

For example, the amplitude of a solitary wave with a 4-min duration propagating offshore from the depth $40 \mathrm{~m}$ and distance of $500 \mathrm{~m}$ from the coast should be more than $100 \mathrm{~m}$.

The behavior of 1- and 2-m height waves propagating seawards is shown in Fig. 2. It can be seen that, practically, there are no nonlinear effects for the wave propagating offshore.

For different applications it is important to know the wave spectrum and its changes. The spectral characteristics of the nonlinear wave propagating in the basin of constant depth are studied in (Zahibo et al., 2008). Spectral representation of the wave moving onshore in a basin of slowly varying depth can be found from Eq. (3). Let us study a transformation of a sine wave with amplitude $A_{0}$ and frequency $\omega\left[\eta_{0}=A_{0} \sin (\omega t)\right]$ along the bottom profile (2). In this case the wave field can be presented as a Fourier series

$\eta(T, x)=\sum_{n=1}^{\infty} A_{n}(x) \sin (n \omega T)$,

where $T=t-\tau(x)$ and

$A_{n}(x)=$

$=\frac{2 A_{0}}{\pi}\left(\frac{x_{0}}{x}\right)^{1 / 3} \int_{0}^{\pi} \sin \left(\omega T+\frac{9 \eta \omega x_{0}}{8 h_{0} \sqrt{g h_{0}}}\left[\left(\frac{x_{0}}{x}\right)^{4 / 3}-1\right]\left(\frac{x}{x_{0}}\right)^{1 / 3}\right) \times$

$\times \sin (n \omega T) d(\omega T)$.

Integral in Eq. (9) can be taken and the final expression for spectral amplitudes is

$A_{n}(x)=$

$=\frac{16 h_{0} \sqrt{g h_{0}}}{9 \omega n x_{0}}\left(\frac{x_{0}}{x}\right)^{1 / 3}\left[\left(\frac{x_{0}}{x}\right)^{4 / 3}-1\right]^{-1} \times$

$\times J_{n}\left(\frac{9 A_{0} \omega n x_{0}}{8 h_{0} \sqrt{g h_{0}}}\left[\left(\frac{x_{0}}{x}\right)^{4 / 3}-1\right]\right)$,

where $J_{n}$ are Bessel functions of the $n$-th order. Using the breaking distance for a sine wave (6) Eq. (10) can be rewritten in the following form:

$$
\begin{aligned}
& A_{n}(x)= \\
& =\frac{2 A_{0}}{n}\left(\frac{x_{0}}{x}\right)^{1 / 3}\left[\frac{\left(\frac{x_{0}}{X_{\mathrm{Br}}}\right)^{4 / 3}-1}{\left(\frac{x_{0}}{x}\right)^{4 / 3}-1}\right] \times \\
& \times J_{n}\left(n\left[\frac{\left(\frac{x_{0}}{x}\right)^{4 / 3}-1}{\left(\frac{x_{0}}{X_{\mathrm{Br}}}\right)^{4 / 3}-1}\right]\right) .
\end{aligned}
$$
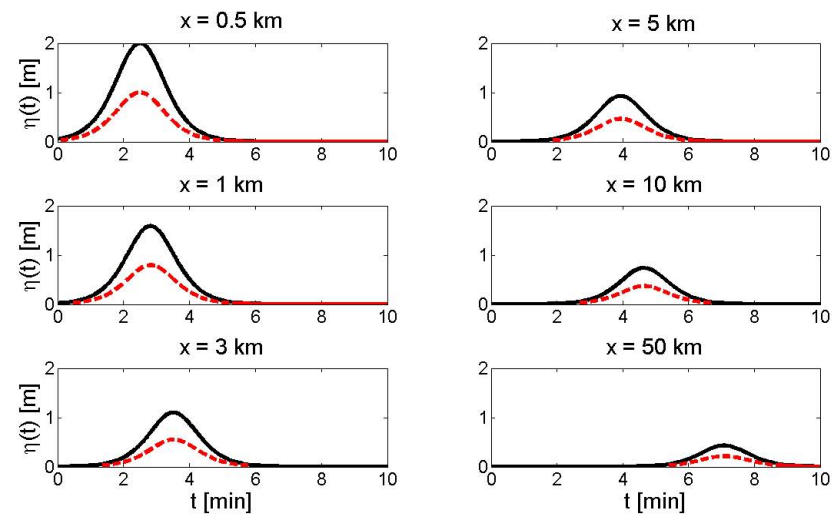

Fig. 2. Time series of nonlinear wave transformation during its propagation seawards; red dashed line corresponds to the wave with $A_{0}=1 \mathrm{~m}$, black solid line - to $A_{0}=2 \mathrm{~m}$.

From Eq. (11) we can find proportions between different harmonics and their changes with the time. In particular, at the moment of the first wave breaking $x=X_{\mathrm{Br}}$, amplitude of the first $A_{1}$ and second $A_{2}$ harmonics are:

$$
\begin{aligned}
& \frac{A_{2}}{A_{0}}=\frac{J_{2}(2)}{2}\left(\frac{x_{0}}{X_{\mathrm{Br}}}\right)^{1 / 3} \approx 0.18\left(\frac{x_{0}}{X_{\mathrm{Br}}}\right)^{1 / 3}, \\
& \frac{A_{1}}{A_{0}}=J_{1}(1)\left(\frac{x_{0}}{X_{\mathrm{Br}}}\right)^{1 / 3} \approx 0.44\left(\frac{x_{0}}{X_{\mathrm{Br}}}\right)^{1 / 3} .
\end{aligned}
$$

It follows from Eq. (12) that at the moment of the first wave breaking amplitude of the second harmonics $A_{2}$ is connected to the first harmonics $A_{1}$ in the following way:

$A_{2}=\frac{A_{1} J_{2}(2)}{2 J_{1}(1)} \approx 0.4 A_{1}$.

The results discussed above have been obtained with the use of the nonlinear WKB approximation valid at large distances $\sim X_{\mathrm{Br}}$ (Engelbrecht et al., 1988). However, if we apply the direct perturbation theory to Eq. (3), found with an assumption of smoothly varying depth (see Didenkulova, 2009 for details), which is formally valid at distances smaller than $X_{\mathrm{Br}}$, in the second order of the perturbation theory the field will consist of two harmonics only:

$$
\begin{aligned}
& \eta=A_{0}\left(\frac{x_{0}}{x}\right)^{1 / 3} \sin (\omega T)+ \\
& +\frac{A_{0}}{2}\left(\frac{x_{0}}{x}\right)^{1 / 3} \frac{\left[\left(\frac{x_{0}}{x}\right)^{4 / 3}-1\right]}{\left[\left(\frac{x_{0}}{X_{\mathrm{Br}}}\right)^{4 / 3}-1\right]} \sin (2 \omega T) .
\end{aligned}
$$

Equation (14) can also be obtained from Eq. (12) assuming $x \ll X_{\mathrm{Br}}$.

The first term in Eq. (14) is a linear solution of shallow water equations along the bottom profile described by Eq. (2) and the second term represents a small nonlinear correction 
to this solution. The amplitude of the correction term is proportional to the squared initial amplitude and changes with the distance as $x^{-5 / 3} \sim h^{-5 / 4}$, which is significantly stronger than the Green's law. So, when the wave approaches the shore the amplitude of the nonlinear correction grows faster than the one of the linear term and the wave tends to break. Of course, this conclusion is valid for both, asymptotic and direct perturbation approaches.

The amplitude of the small nonlinear correction $A_{2}$ with respect to the amplitude of the linear term $A_{1}$ calculated from Eq. (14) can be expressed as a function of the only parameter $\frac{X_{\mathrm{Br}}}{x_{0}}$

$\frac{A_{2}}{A_{1}}=\frac{1}{2} \frac{\left[\left(\frac{x_{0}}{x}\right)^{4 / 3}-1\right]}{\left[\left(\frac{x_{0}}{X_{\mathrm{Br}}}\right)^{4 / 3}-1\right]}$,

and it is shown in Fig. 3. It can be seen from Eq. (15) that at the breaking distance the amplitude of the second harmonics $A_{2}$ is twice smaller than the amplitude of the first harmonics $A_{1}$. Thus, in the vicinity of the wave breaking the perturbation theory overestimates the amplitude of the second harmonics by $20 \%$ compare to the asymptotic approach (13).

Similar analysis can be performed for the wave propagating seawards. In this case amplitudes of harmonics, obtained with the use of asymptotic approach Eqs. (11), and the wave field found from the perturbation theory Eq. (14), should be re-written as

$$
\begin{aligned}
& A_{n}^{\text {off }}(x)=\frac{2 A_{0}}{n}\left(\frac{x_{0}}{x}\right)^{1 / 3}\left[\frac{\left(\frac{x_{0}}{X_{\mathrm{Br}}^{\text {off }}}\right)^{4 / 3}-1}{\left(\frac{x_{0}}{x}\right)^{4 / 3}-1}\right] \times \\
& \times J_{n}\left(n\left[\frac{\left(\frac{x_{0}}{x}\right)^{4 / 3}-1}{\left(\frac{x_{0}}{X_{\mathrm{Br}}^{\text {off }}}\right)^{4 / 3}-1}\right]\right),
\end{aligned}
$$

$\eta^{\mathrm{off}}=A_{0}\left(\frac{x_{0}}{x}\right)^{1 / 3} \sin (\omega T)+$

$+\frac{A_{0}}{2}\left(\frac{x_{0}}{x}\right)^{1 / 3} \frac{\left[\left(\frac{x_{0}}{x}\right)^{4 / 3}-1\right]}{\left[\left(\frac{x_{0}}{X_{\mathrm{Br}}^{\text {off }}}\right)^{4 / 3}-1\right]} \sin (2 \omega T)$.

It can be seen that in the case of the wave propagating seawards the perturbation theory in the vicinity of the wave breaking also overestimates the amplitude of the second harmonics compare to the asymptotic approach.

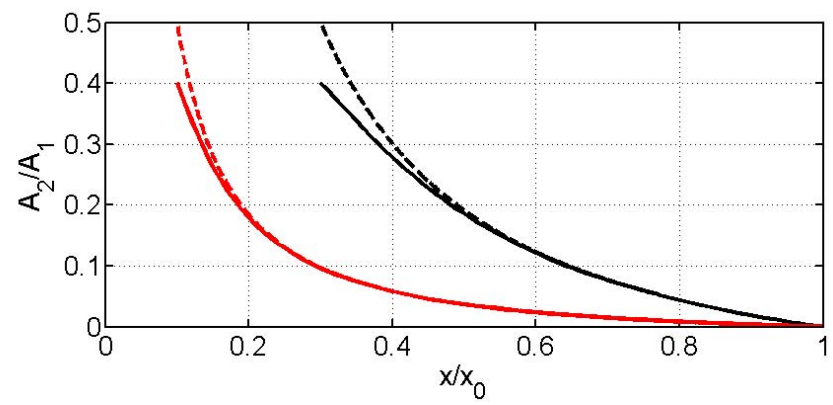

Fig. 3. Amplitude of the second harmonic $A_{2}$ with respect to the amplitude of the first harmonic $A_{1}$ calculated from Eq. (14) for $\frac{X_{\mathrm{Br}}}{x_{0}}=0.1$ (red dashed line) and 0.3 (black dashed line); solid lines show the corresponding ratio of $A_{2}$ and $A_{1}$ calculated from Eq. (11).

\section{Non-adiabatic nonlinear effects at the non-reflecting beach}

In the general case when the water depth is varied arbitrary, we cannot find neither asymptotic no exact analytical solution of Eqs. (1); this also applies to the non-reflecting beach (2). That is why here in order to describe the nonlinear effects at the non-reflecting beach of an arbitrary slope we use a direct perturbation theory. We should also note that based on the analysis presented in the previous section we expect that the perturbation theory will overestimate the magnitude of the nonlinear correction by up to $20 \%$.

Let us assume a weakly nonlinear case of Eqs. (1)

$\eta=\eta_{1}+\eta_{\mathrm{nl}}, \quad u=u_{1}+u_{\mathrm{nl}}$,

$\eta_{\mathrm{nl}} \ll \eta_{1}, \quad u_{\mathrm{nl}} \ll u_{1}$,

where $\eta_{1}$ and $u_{1}$ correspond to the water displacement and flow velocity in the linear case and $\eta_{\mathrm{nl}}$ and $u_{\mathrm{nl}}$ reflect the influence of the nonlinearity.

When substituting Eq. (18) into Eq. (1) we obtain linear shallow water equations for $\eta_{1}$ and $u_{1}$ to a first approximation:

$\frac{\partial \eta_{1}}{\partial t}+\frac{\partial}{\partial x}\left[h(x) u_{1}\right]=0, \quad \frac{\partial u_{1}}{\partial t}+g \frac{\partial \eta_{1}}{\partial x}=0$,

and for small nonlinear corrections $\eta_{\mathrm{nl}}$ and $u_{\mathrm{nl}}$ we have the system of linear inhomogeneous equations

$\frac{\partial \eta_{\mathrm{nl}}}{\partial t}+\frac{\partial}{\partial x}\left[h(x) u_{\mathrm{nl}}\right]=-\frac{\partial}{\partial x}\left[\eta_{1} u_{1}\right]$,

$\frac{\partial u_{\mathrm{nl}}}{\partial t}+g \frac{\partial \eta_{\mathrm{nl}}}{\partial x}=-\frac{1}{2} \frac{\partial u_{1}^{2}}{\partial x}$.

Equation (21) can be reduced to the inhomogeneous wave equation for the water displacement:

$\frac{\partial^{2} \eta_{\mathrm{nl}}}{\partial t^{2}}-\frac{\partial}{\partial x}\left[g h(x) \frac{\partial \eta_{\mathrm{nl}}}{\partial x}\right]=\Phi(x, t)$,
$\Phi(x, t)=\frac{1}{2} \frac{\partial}{\partial x}\left[h(x) \frac{\partial u_{1}^{2}}{\partial x}\right]-\frac{\partial^{2}}{\partial t \partial x}\left[\eta_{1} u_{1}\right]$. 
For the bottom profile defined by Eq. (2) the general solution of the homogeneous Eq. (22) represents a superposition of two travelling waves propagating in opposite directions. Here we consider only one wave propagating shoreward, presented in the complex form (Didenkulova et al., 2009)

$\eta_{1}(x, t)=A_{1}(x) e^{i \omega t}+A_{1}^{*}(x) e^{-i \omega t}$,

$u_{1}(x, t)=U_{1}(x) e^{i \omega t}+U_{1}^{*}(x) e^{-i \omega t}$,

where

$A_{1}(x)=\frac{A_{0}}{2}\left[\frac{h_{0}}{h(x)}\right]^{1 / 4} e^{i \omega \tau(x)}$
$U_{1}(x)=\frac{A_{0}}{2} \sqrt{\frac{g}{h(x)}}\left[\frac{h_{0}}{h(x)}\right]^{1 / 4}\left[-1+\frac{\sqrt{g h}}{4 h i \omega} \frac{d h}{d x}\right] e^{i \omega \tau(x)}$

Substituting Eq. (24) into Eq. (22) in the right hand side of Eq. (22) we have

$\Phi(x, t)=\Phi_{10}(x, t)+\Phi_{11}(x, t) e^{2 i \omega t}+\Phi_{11}^{*}(x, t) e^{-2 i \omega t}$,

where

$$
\begin{aligned}
& \Phi_{10}(x, t)=\frac{d}{d x}\left(h \frac{d\left|U_{1}\right|^{2}}{d x}\right), \\
& \Phi_{11}(x, t)=\frac{1}{2} \frac{d}{d x}\left(h \frac{d\left(U_{1}^{2}\right)}{d x}\right)-2 i \omega \frac{d\left(A_{1} U_{1}\right)}{d x} .
\end{aligned}
$$

Since the forcing function has three different frequencies then the solution of Eq. (22) should also consists of three terms

$\eta_{\mathrm{nl}}(x, t)=\eta_{10}(x)+\eta_{11}(x) e^{2 i \omega t}+\eta_{11}^{*} e^{-2 i \omega t}$.

Substituting Eq. (26) into Eq. (22) we obtain two ordinary differential equations for definition of $\eta_{10}(x)$ and $\eta_{11}(x)$ (equation for $\eta_{11}^{*}(x)$ is obtained automatically)

$\frac{d}{d x}\left[g h(x) \frac{d \eta_{10}}{d x}\right]=-\frac{d}{d x}\left[h(x) \frac{d}{d x}\left|U_{1}(x)\right|^{2}\right]$,

$\frac{d}{d x}\left[g h(x) \frac{d \eta_{11}}{d x}\right]+4 \omega^{2} \eta_{11}=$

$=-\left[\frac{1}{2} \frac{d}{d x}\left(h \frac{d U_{1}^{2}}{d x}\right)-2 i \omega \frac{d\left(A_{1} U_{1}\right)}{d x}\right]$.

Eq. (27) describes the set-up and set-down effects and can be integrated

$\eta_{10}(x)=C_{1}+\int \frac{C_{2} d x}{g h(x)}-\frac{\left|U_{1}(x)\right|^{2}}{g}, C_{1}=\frac{\left|U_{1}\left(x=x_{0}\right)\right|^{2}}{g}$,

$C_{2}=0$,

coefficients $C_{1}$ and $C_{2}$ are chosen to satisfy the condition of no water disturbances before wave arrival and after the wave passage. Equation (28) is the inhomogeneous second-order

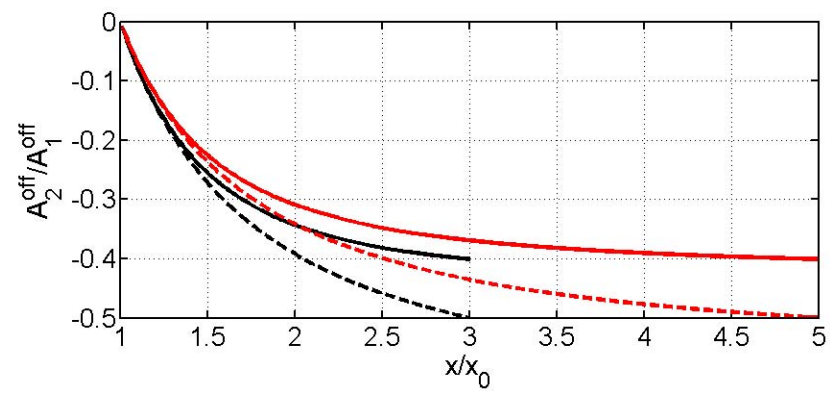

Fig. 4. Amplitude of the nonlinear correction $A_{2}$ with respect to the amplitude of the linear term $A_{1}$ calculated from Eq. (17) for $\frac{X_{\mathrm{Br}}^{\mathrm{off}}}{x_{0}}=$ 3 (black dashed line) and 5 (red dashed line); solid lines show the corresponding ratio of $A_{2}$ and $A_{1}$ calculated from Eq. (16).

differential equation with variable coefficients, which can be solved by the method of variation of constants. Let us seek the solution of Eq. (28) in the following form:

$\eta_{11}=H_{1}(x)\left[\frac{x_{0}}{x}\right]^{1 / 3} e^{2 i \omega \tau(x)}+H_{2}(x)\left[\frac{x_{0}}{x}\right]^{1 / 3} e^{-2 i \omega \tau(x)}$

where $\tau$ is a travel time from $x_{0}$ and $H_{1}(x)$ and $H_{2}(x)$ are unknown functions of $x$. Substituting Eq. (30) into Eq. (28) we obtain a system for variable amplitudes $H_{1}(x)$ and $H_{2}(x)$ :

$\left\{\begin{array}{l}\frac{d H_{1}(x)}{d x} e^{2 i \omega \tau(x)}+\frac{d H_{2}(x)}{d x} e^{-2 i \omega \tau(x)}=0 \\ \frac{d H_{1}(x)}{d x} e^{2 i \omega \tau(x)}-\frac{d H_{2}(x)}{d x} e^{-2 i \omega \tau(x)=} \\ =-\frac{A_{0}^{2} g}{8 i \omega \sqrt{g h_{0}}}\left[\frac{x_{0}^{2 / 3}}{x^{8 / 3}}-\frac{32 \sqrt{g h_{0}}}{27 i \omega x^{3}}-\frac{28 g h_{0}}{81 \omega^{2} x^{10 / 3} x_{0}^{2 / 3}}-\right. \\ \left.-\frac{i \omega x_{0}^{4 / 3}}{3 \sqrt{g h_{0}} x^{7 / 3}}+\frac{3 \omega^{2} x_{0}^{2}}{g h_{0} x^{2}}\right] e^{2 i \omega \tau(x)}\end{array}\right.$

from which $H_{1}(x)$ and $H_{2}(x)$ can be found. In particular,

$$
\begin{aligned}
& H_{1}(x)= \\
& \frac{A_{0}^{2} g}{16 i \omega \sqrt{g h_{0}}}\left[\frac{x_{0}}{2 x^{2}}-\frac{32 x_{0}^{1 / 3} \sqrt{g h_{0}}}{63 i \omega x^{7 / 3}}-\frac{7 g h_{0}}{54 \omega^{2} x^{8 / 3} x_{0}^{1 / 3}}-\frac{i \omega x_{0}^{5 / 3}}{5 \sqrt{g h_{0}} x^{5 / 3}}+\frac{9 \omega^{2} x_{0}^{7 / 3}}{4 g h_{0} x^{4 / 3}}\right]- \\
& -\frac{A_{0}^{2} g}{16 i \omega \sqrt{g h_{0}}}\left[\frac{1}{2 x_{0}}-\frac{32 \sqrt{g h_{0}}}{63 i \omega x^{2}}-\frac{7 g h_{0}}{54 \omega^{2} x_{0}^{3}}-\frac{i \omega}{5 \sqrt{g h_{0}}}+\frac{9 \omega^{2} x_{0}}{4 g h_{0}}\right]
\end{aligned}
$$

and describes a part of second harmonics of the wave propagating shoreward. At large distances Eq. (32) behaves like:

$H_{1}(x) \sim-\frac{9 i \omega A_{0}^{2} x_{0}}{64 h_{0} \sqrt{g h_{0}}}\left[\frac{x_{0}^{4 / 3}}{x^{4 / 3}}-1\right]$,

which corresponds to $1 / 4$ of the amplitude of the second harmonics found for the wave propagation along the bottom of slowly varying depth using the perturbation theory (Eq. 14), and together with $H_{1}^{*}(x)$, which is contained in $\eta_{11}^{*}(x)$ (Eq. 26), they will give a half of it.

In a similar way $H_{2}(x)$ can be found from Eq. (31) in the integral form

$H_{2}(x)=\frac{A_{0}^{2} g}{16 i \omega \sqrt{g h_{0}}} \times$ 


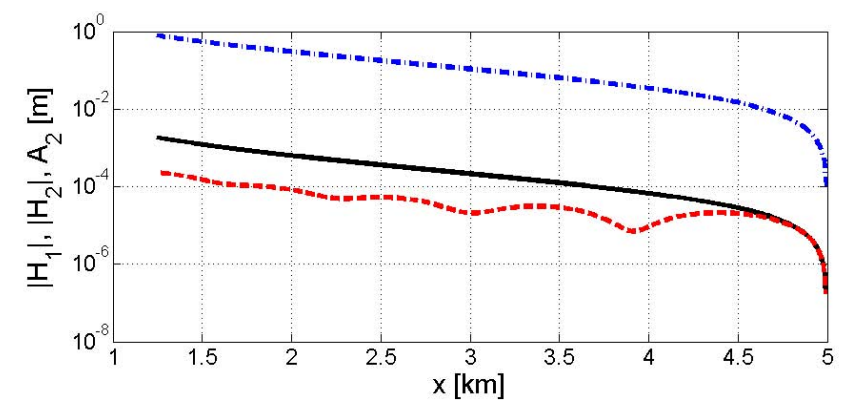

Fig. 5. Absolute values of $H_{1}(x)$ (black solid line) and $H_{2}(x)$ (red dashed line) for 1-meter wave propagating shoreward. Blue dashdotted line corresponds to the amplitude of the second harmonics found for the wave propagation along the bottom of slowly varying depth using the perturbation theory Eq. (14).

$$
\begin{aligned}
& \times \int_{x_{0}}^{x}\left[\frac{x_{0}}{x^{3}}-\frac{32 x_{0}^{1 / 3} \sqrt{g h_{0}}}{27 i \omega x^{10 / 3}}-\right. \\
& \left.-\frac{28 g h_{0}}{81 \omega^{2} x^{11 / 3} x_{0}^{1 / 3}}-\frac{i \omega x_{0}^{5 / 3}}{3 \sqrt{g h_{0}} x^{8 / 3}}+\frac{3 \omega^{2} x_{0}^{7 / 3}}{g h_{0} x^{7 / 3}}\right] e^{4 i \omega \tau(x)} d x
\end{aligned}
$$

It contains components of both incoming and reflected waves, which cannot be separated, and, therefore, describes the nonlinear reflection from the beach which is neglected in the linear approximation.

Both amplitudes $H_{1}(x)$ and $H_{2}(x)$ are displayed in Fig. 5 for 1-m wave propagating shoreward, which is initially located at the distance $5-\mathrm{km}$ from the coast at the $40 \mathrm{~m}$ water depth. For convenience of demonstration Fig. 5 is shown in semi-logarithmic scale.

It can be seen in Fig. 5 that $H_{2}(x)$ has a quasi-periodic structure, which confirms the existence of both incident and reflected components in $H_{2}(x)$. Decreasing depth results in the decrease in the wavelength and spatial period of oscillation when the wave approaches the shore. These changes are close to $\sqrt{h}$ as it could be expected from WKB approach. Since amplitude of waves increases significantly in the coastal zone (in two orders, see Fig. 5) small oscillations are vanished against this background. Amplitude of the second harmonics found for the wave propagation along the bottom of slowly varying depth using the perturbation theory Eq. (14) is larger than sum of $H_{1}(x)$ and $H_{2}(x)$.

Similar calculations are performed for the 1-m wave propagating offshore from the initial distance from the coast of $500 \mathrm{~m}$ and the water depth of $40 \mathrm{~m}$. Corresponding amplitudes $H_{1}(x)$ and $H_{2}(x)$ are shown in Fig. 6.

Comparison of the second harmonics found from the nonlinear WKB approximation (10) and nonlinear term (24) is shown in Figs. 7 and 8 for 1-m sine wave propagating shoreward and offshore respectively. The wave with a period of $4 \mathrm{~min}$ is initially located $5 \mathrm{~km}$ from the coast $(500 \mathrm{~m}$ for the wave propagating seawards) at the water depth $40 \mathrm{~m}$. As in

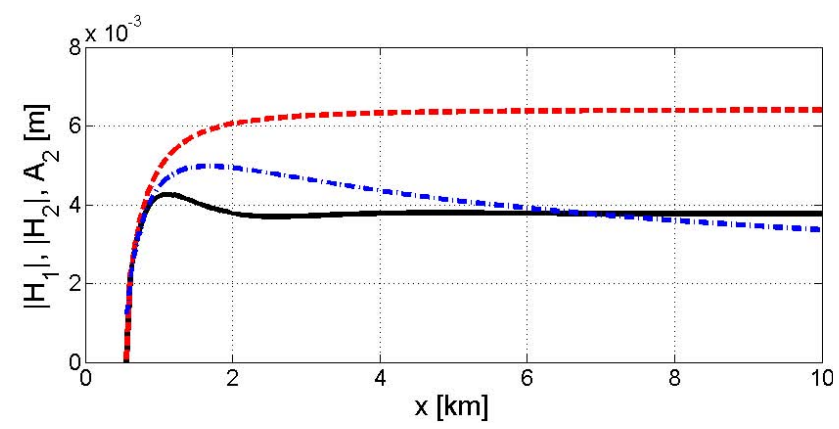

Fig. 6. $H_{1}(x)$ (black solid line) and $H_{2}(x)$ (red dashed line) for 1 -meter wave propagating offshore. Blue dash-dotted line corresponds to the amplitude of the second harmonics found for the wave propagation along the bottom of slowly varying depth using the perturbation theory (14).

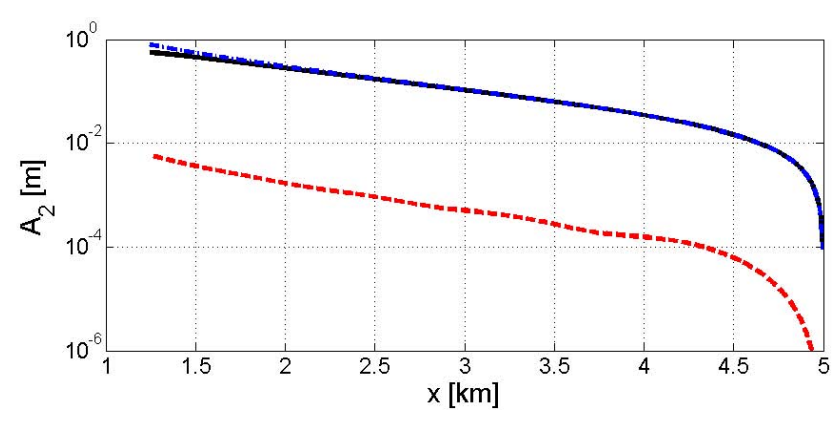

Fig. 7. Comparison of amplitudes of the second harmonics found from the nonlinear WKB approximation (10) (black solid line) and nonlinear term (26) (red dashed line) for the wave propagating shoreward. Blue dash-dotted line corresponds to the amplitude of the second harmonics found for the wave propagation along the bottom of slowly varying depth using the perturbation theory (14).

Fig. 5 it is more convenient to use semi-logarithmic axis in Fig. 7.

It can be seen that amplitude of the second harmonics for the wave propagating shoreward in the perturbation theory is smaller than the one predicted by nonlinear WKB approach (Fig. 7). Hence, at the same distance waves are less nonlinear within the perturbation theory than in the WKB approximation Eq. (10). This may be explained by the presence of the reflected wave, which is significant near the coast. It decreases the total amplitude of the wave and, therefore, decreases its nonlinearity. Due to the strong wave amplification in the nearshore region, oscillations related to the interaction of incident and reflected waves are weakly visible in Fig. 7, while they are well seen in Fig. 5. However, for the wave moving offshore second harmonics predicted by both perturbation theory and nonlinear WKB approximation decay with the distance from the coast and have the same asymptotic (Fig. 8). Oscillations reflect the influence of the wave reflection which is very well seen in deep water due to minor 


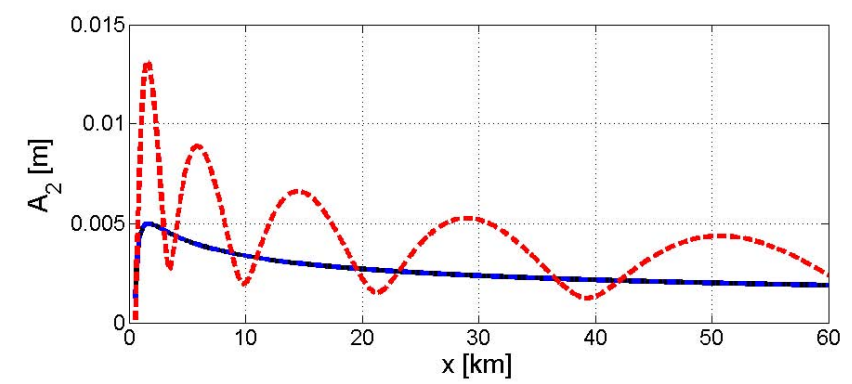

Fig. 8. Comparison of amplitudes of the second harmonics found from the nonlinear WKB approximation (10) (black solid line) and nonlinear term (26) (red dashed line) for the wave propagating offshore. Blue dash-dotted line corresponds to the amplitude of the second harmonics found for the wave propagation along the bottom of slowly varying depth using the perturbation theory (14).

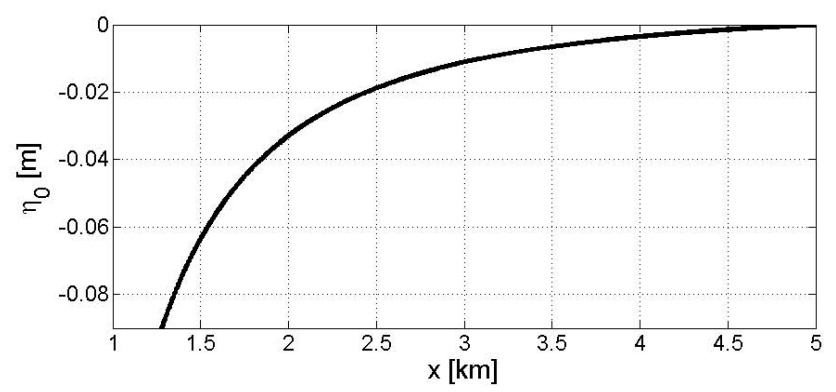

Fig. 9. Wave set-down for the wave propagating shoreward.

nonlinear effects. Variations of the amplitude of the second harmonics in deep water are weak and wave is almost linear.

In conclusion let us discuss the effects of wave set-up and set-down (zero harmonics) found from the direct perturbation theory (Eq. 29). Its changes for the 1-m wave propagating shoreward from $5 \mathrm{~km}$ distance from the coast $(40 \mathrm{~m}$ depth) are shown in Fig. 9. It can be seen that during wave propagation to the coast before the wave breaking, the mean sea level decreases, which leads to the known effect of wave set-down outside the wave breaking zone (Longuet-Higgins and Stewart, 1963; Bowen et al., 1968; Dean and Walton, 2009).

At the same time during wave propagation seaward the mean sea level rises, but those effects are almost negligible. For example, it is shown in Fig. 10 that the seaward propagation of 1-m wave from the initial location $5 \mathrm{~km}$ (depth $40 \mathrm{~m}$ ) results in less than $1 \mathrm{~cm}$ set-up at the distance $50 \mathrm{~km}$ from the coast. It also follows from Eq. (29) that the wave set-up for the wave propagation to deep water has an asymptotic and is limited by

$\eta_{0}(x \rightarrow \infty) \rightarrow \frac{\left|U_{1}\left(x=x_{0}\right)\right|^{2}}{g}$.

For example, shown in Fig. 10 the maximum set-up is only $8 \mathrm{~mm}$.

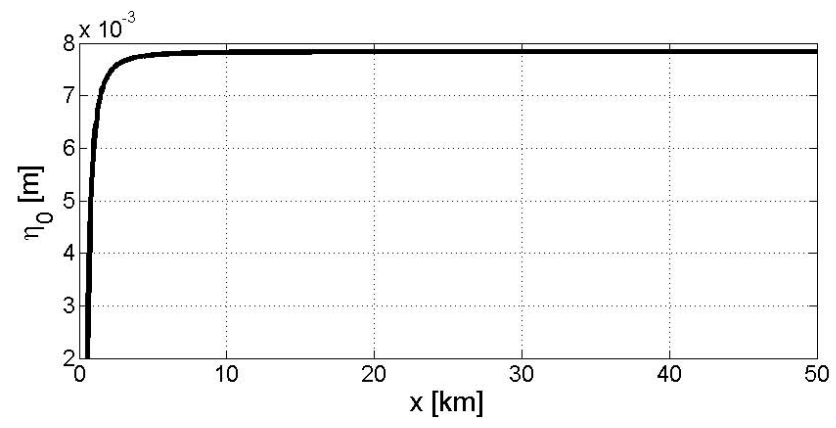

Fig. 10. Wave set-up for the wave propagating seaward.

\section{Conclusions}

Nonlinear evolution of long waves above special bottom profile $\left(h \sim x^{4 / 3}\right)$ is studied. This profile has a special interest since it allows non-reflecting wave propagation within the linear shallow-water theory; the shape of the wave during its propagation along such a beach is also conserved in time (Didenkulova et al., 2009). At the same time in the nonlinear theory reflection from such a beach exists as it is demonstrated here with the use of the perturbation theory.

It is shown in the framework of the nonlinear WKB approach assuming slow bottom changes and weak nonlinearity that nonlinearity leads to the wave breaking near the coast. In contrary, when the wave propagates seawards it may break only if it is of unrealistically large amplitude. Using direct perturbation theory the generation of zero- and second- harmonics is studied for an arbitrary slope of non-reflecting geometry. It is demonstrated that in the second-order of the perturbation theory the nonlinearity leads to the wave reflection and change in the sea level. The mean sea level decreases during wave propagation shoreward, which leads to the wellknown effect of wave set-down before wave breaking (Dean and Walton, 2009). For the seaward-going wave the mean sea level change is minor and limited by the value, depending on the water flow velocity at the initial location.

The variations of the second harmonics for the seawardsgoing wave are also weak, demonstrating almost linear wave behavior. It oscillates around values of the second harmonics predicted by the nonlinear WKB approximation. At the same time for the wave propagating shoreward the nonlinear effects become important. The WKB approximation overestimates the second harmonics, which may be explained by the presence of the reflected wave, which is significant near the coast. This also results that the wave breaks closer to the shore than it is predicted by nonlinear WKB approximation. 
Acknowledgements. Partial support from the targeted financing by the Estonian Ministry of Education and Research (grant SF0140007s11), Estonian Science Foundation (grant 8870), RFBR (Grants 11-05-00216, 11-05-92002 and 11-02-00483) and Russian president grant $(\mathrm{M} \mathcal{K}-1440.2012 .5)$ is greatly acknowledged.

\section{Edited by: R. Grimshaw}

Reviewed by: two anonymous referees

\section{References}

Bjorkavag, M. and Kalisch, H.: Wave breaking in Boussinesq models for undular bores, Phys. Lett. A, 375, 1570-1578, 2011.

Bowen, A. J., Inman, D. L., and Simmons, V. P.: Wave set-down and wave set-up, J. Geophys. Res., 73, 2569-2577, 1968.

Caputo, J.-G. and Stepanyants, Y. A.: Bore formation, evolution and disintegration into solitons in shallow inhomogeneous channels, Nonlin. Processes Geophys., 10, 407-424, doi:10.5194/npg-10407-2003, 2003.

Carrier, G. F. and Greenspan, H. P.: Water waves of finite amplitude on a sloping beach, J. Fluid Mech., 4, 97-109, 1958.

Dean, R. G. and Dalrymple, R. A.: Coastal processes with engineering applications, Cambridge University Press, 475 pp., 2002.

Dean, R. G. and Walton, T. L.: Wave setup, in: Handbook of coastal and ocean engineering, edited by: Kim, Y. C., World Sci., Singapore, 2009.

Didenkulova, I.: Nonlinear long-wave deformation and runup in a basin of varying depth, Nonlin. Processes Geophys., 16, 23-32, doi:10.5194/npg-16-23-2009, 2009.

Didenkulova, I. and Pelinovsky, E.: Non-dispersive traveling waves in strongly inhomogeneous water channels, Phys. Lett. A, 373, 3883-3887, doi:10.1016/j.physleta.2009.08.051, 2009.

Didenkulova, I. and Pelinovsky, E.: Traveling water waves along a quartic bottom profile, Proc. Estonian Ac. Sci., 59, 166-171, doi:10.3176/proc.2010.2.16, 2010.

Didenkulova, I. and Pelinovsky, E.: Runup of tsunami waves waves in U-shaped bays, Pure Appl. Geophys., 168, 1239-1249, doi:10.1007/s00024-010-0232-8, 2011.

Didenkulova, I. and Soomere, T.: Formation of two-section cross-shore profile under joint influence of random short waves and groups of long waves Mar. Geol., 289, 29-33, doi:10.1016/j.margeo.2011.09.011, 2011.

Didenkulova, I., Pelinovsky, E., and Soomere, T: Runup Characteristics of Symmetrical Solitary Tsunami Waves of "Unknown" Shapes, Pure Appl. Geophys., 165, 2249-2264, doi:10.1007/s00024-008-0425-6, 2008.

Didenkulova, I., Pelinovsky, E., and Soomere, T.: Long surface wave dynamics along a convex bottom, J. Geophys. Res.-Oceans, 114, C07006, doi:10.1029/2008JC005027, 2009.
Didenkulova, I., Nikolkina, I., Pelinovsky, E., and Zahibo, N.: Tsunami waves generated by submarine landslides of variable volume: analytical solutions for a basin of variable depth, Nat. Hazards Earth Syst. Sci., 10, 2407-2419, doi:10.5194/nhess-102407-2010, 2010.

Engelbrecht, J. K., Fridman, V. E., and Pelinovsky, E. N.: Nonlinear Evolution Equations, Pitman Research Notes in Mathematics Series, No. 180, Longman, London, 1988.

Gurtin, M. E.: On the breaking of water waves on a sloping beach of arbitrary shape, Quart. Appl. Math., 33, 187-189, 1975.

Kowalik, Z. and Murty, T. S.: Numerical Modelling of Ocean Dynamics, Advanced Series on Ocean Engineering, World Scientific, Singapore, Vol. 5, 1993.

Liu, P. L.-F., Yeh, H., and Synolakis, C.: Advances in Coastal and Ocean Engineering Advanced Numerical Models for Simulating Tsunami Waves and Runup, World Scientific, 2008.

Longuet-Higgins, M. S. and Stewart, R. W.: A note on wave set-up, J. Mar. Res., 21, 4-10, 1963.

Massel, S. R.: Hydrodynamics of coastal zones. Elsevier, Amsterdam, 1989.

Mei, C. C.: Applied dynamics of ocean surface waves, Singapore, World Scientific, 1989.

Nwogu, O. G.: Numerical prediction of breaking waves and currents with a Boussinesq model, Proc. 25th International Conference on Coastal Engineering, ICCE'96, Orlando, 4807-4820, 1996.

Raubenneimer, B. and Guza, R. T.: Observations and predictions of run-up, J. Geophys. Res., 101, 25575-25587, 1996.

Raubenneimer, B., Guza, R. T., and Elgar, S.: Field observations of wave-driven setdown and setup, J. Geophys. Res., 106, 46394638, 2001.

Sato, S. and Kabiling, M. B.: A numerical simulation of beach evolution based on a nonlinear dispersive wave-current model. Proceedings of the 24th International Conference held in Kobe, Japan, Coastal Engineering 1994, New York, 2557-2570, 1995.

Sorensen, R. M.: Basic Wave Mechanics, Wiley, New York, 1993.

Synolakis, C. E.: The runup of solitary waves, J. Fluid Mech., 185, 523-545, 1987.

Varley, E., Venkataraman, R., and Cumberbatch, E.: The propagation of large amplitude tsunamis across a basin of changing depth, I. Off-shore behaviour, J. Fluid Mech., 49, 775-801, 1971.

Yeh, H., Liu, P. L.-F., and Synolakis, C.: Long-Wave Runup Models, World Scientific, 1996.

Zahibo, N., Didenkulova, I., Kurkin, A., and Pelinovsky, E.: Steepness and spectrum of nonlinear deformed shallow water wave, Ocean Eng., 35, 47-52, doi:10.1016/j.oceaneng.2007.07.001, 2008. 